\title{
The method of calculating the number of truck stops
}

\author{
Anatoliy Dubolazov ${ }^{1}$, Nikita Lukashevich ${ }^{1}$, Zoya Simakova ${ }^{1}$, Egor Temirgaliev $^{1, *}$ \\ ${ }^{1}$ Peter the Great St.Petersburg Polytechnic University, Polytechnicheskaya, 29, St. Petersburg, \\ 195251, Russia
}

\begin{abstract}
A review of the theoretical foundations of the main approaches to the description of traffic flows has been carried out. A key parameter is proposed for comparing costs when driving on ordinary roads and highspeed toll roads. The possibilities for determining the quantitative assessment of this parameter using the tools of the theory of traffic flows are considered. A new technique for solving the task with the help of modern information technologies is proposed. Additional parameters are identified, and measures are indicated for further work and improvement of the proposed method.
\end{abstract}

\section{Introduction}

The basic idea of sustainable development is to meet modern consumption needs so that future generations will be able to satisfy their requirements. Planet Earth can be considered a closed system. If in one place something has arrived, then in another has gone. The only incoming flow from outside this system is solar energy. To satisfy needs it is necessary to follow the basic principles of sustainable development of logistics [1, 2]:

- Efficient use of energy resources.

- Efficient use of raw materials and material resources.

- Raw materials loss minimization as well as loss minimization of materials, products and energy that are used for production, distribution and management of various streams after the consumption of products.

- Effective planning and the capacity building of the logistics system.

- Timely modernization of the existing capacity of the logistics system.

- Minimization of the environmental impact on the environment.

One of the main environmental pollutants is transport. The amount of direct annual damage from the functioning of the transport complex of Russia is more than $\$ 3.5$ billion. More than half of the environmental damage comes from passenger traffic. Their share in environmental damage from air and road transport is especially high. Passenger traffic dominates in air transport; therefore, their impact on the total environmental damage exceeds $80 \%$. The share of environmental damage from the impact of road transport is approximately $63 \%$, including $46 \%$ in passenger traffic, as cars dominate the structure of the car fleet (out of 39 million vehicles in Russia, about 33 million are cars, 5.3 trucks and 896 thousand -

\footnotetext{
* Corresponding author: temirgaliev@kafedrapik.ru
} 
buses $[3,4])$. On other types of transport, the main environmental damage is caused during freight traffic due to their greater share in the transportation structure [5].

Transport is one of the main air pollutants. On average in Russia, the share of transport in air pollution is $40-45 \%$, and in large cities it is up to $90 \%$. At least 15 million citizens are exposed to the risk of health loss due to air pollution from transport emissions. The share of transport emissions in air pollution is higher than the share of any industry. In the second place in terms of emissions into the atmosphere is energy, then non-ferrous and ferrous metallurgy. Next, the oil-producing, oil-refining, machine-building, gas, and other industries are located in decreasing order. At the same time, the reduction in total emissions for all sectors of the economy should be noted. In recent decades, there has been an intensive increase in the number of motor vehicles (from 25 million units in 2001 to 42.4 million units in 2018) [5]. But, despite this trend, since 2006, a reduction in the mass of pollutant emissions began ( 15 thousand tons in 2006, 12 thousand tons in 2018), which is due to the gradual fleet replacement and improvement of the "ecological" structure of the park [5].

Air pollution from mobile sources occurs as a result of fuel combustion. The chemical composition of emissions depends on the type and quality of fuel, production technology, method of combustion in the engine and its technical condition.

The most unfavorable modes of operation are low speeds and "idling" of the engine, when pollutants are released into the atmosphere in quantities far exceeding the emission under load conditions. During the acceleration period, the emission of soot into the atmosphere increases. The technical condition of the engine directly affects the environmental performance of emissions.

Preventing or minimizing the negative consequences of the impact of vehicles on the environment during the course of operation can be achieved through practical measures of various kinds:

- Organizational measures (development of rational traffic routes, the allocation of special lanes for public transport, etc.).

- Technical and technological measures.

- Design activities (improvement of samples of vehicles and their engines, ensuring the elimination or reduction to a minimum negative impact on the environment).

Until 2030 measures implementation in the road complex is also provided in the transport strategy of the Russian Federation [6]:

- Development of a network of public roads and the increasing of their capacity.

- Development and implementation of specialized hydro-meteorological services, improvement of forecasting methods and operational accounting of changing weather conditions, allowing to move from anti-glare control to its prevention.

- Equipping highways of Federal significance with engineering means of protecting the environment from harmful effects in order to reduce the level of noise exposure and pollution of adjacent territories, installation of noise protection screens.

- Development and introduction of new structures (overpasses, tunnels), materials, technologies that will reduce dust generation, prevent water erosion and disturbance_of natural landscapes.

- The Increasing use of fuel- efficient vehicles with lower engine fuel consumption.

- Improving the technical level of vehicles.

- Strengthening control over the technical condition of vehicles in operation according to environmental indicators, limiting emissions of climate gases and recycling of transport organizations.

- Transition to world environmental standards for fuel consumption.

- The transferring of $50 \%$ of automotive vehicles in major cities to alternative fuels. 


\section{Materials and Methods}

From all the above, for intercity road freight transport, the following most significant activities can be distinguished:

- Improving the road network.

- Perfection of vehicle fleet performance.

Today, the state of the roads in the Russian Federation is good. But the main problem remains a large number of roads that connect large cities and pass through a large number of small settlements, in which the traffic flow has to be slowed down, stop at regulated intersections of roads, and traffic jams are possible. An alternative is the rapidly growing network of toll high-speed roads. But, as practice shows, truckers are reluctant to use toll highways. Freight carriers, when using toll roads, have additional costs, that is why in most cases they try to build routes without toll sections [7, 8]. But the authors of this article consider this situation to be ambiguous. So, they set out to clarify and simplify the calculation of costs when using different routes of transportation of goods. According to their opinion costs consist of:

- The cost of fuel and lubricants.

- Worn tire wheels of cars.

- The Plato Toll Collection System (this system was created to ensure compliance with the procedure established by current legislation for charging compensation for damages caused to federal public roads by vehicles with an authorized maximum weight exceeding 12 tons).

- Fare on a paid section of the road.

It should be noted that when driving on a paid section of the road, the fee in the Plato system is not charged, the movement is more uniform, there are no settlements on the way, which leads to a significant decrease of number of sections where cars accelerate or break.

There are many methods for calculating the fuel consumption of a truck when driving in different modes. But none of them offers a way to account the number of sections of braking, acceleration or car stops.

In this paper, the authors propose a solution to this problem with the use of modern information technology and information available to absolutely everyone. Each carrier has the opportunity to use this technique and has access to all the necessary information for making calculations.

The most completely the behaviour of a car in a traffic flow is considered in the theory of traffic flows [9]. In this theory, the characteristics of traffic flows, traffic flow models, methods of applying models in the designing and organization of traffic are studied. To put forward new proposals, it is necessary to consider the main provisions of the existing theory of traffic flows.

Two approaches have emerged in traffic modeling: deterministic and probabilistic (stochastic) [9].

Deterministic models suggest a functional relationship between calculation traffic indicators. In stochastic models, the traffic flow is considered as a probabilistic process.

Regularities in the movement of cars exist, but it is almost impossible to take into account the law of each car movement, hence the need of simplified laws that can be borrowed from mathematical statistics appear.

Traffic flow models can be divided into three groups [9] - analogue models, leader followup models which is related to the deterministic approach, and probabilistic models.

In analogue models, an analogy between the flow of motion of a substance and the transport flow is used - in this case, the laws of gas and hydrodynamics can be applied to the transport flow. 
It is known that a fluid can have a laminar and turbulent flow. In the laminar flow, the fluid moves through the pipeline without mixing, that is, consists of separate independent flows - "elementary streams". In a turbulent flow, the fluid is intensively mixed, which causes additional dynamic phenomena in the pipeline, sometimes accompanied by noise. It is also known that the laminar flow of a fluid is observed at low speeds, whereas with a turbulent flow the flow velocity is higher. To estimate the boundary between laminar and turbulent flow the Reynolds number is used. If we draw an analogy, the traffic without jam on the road can be compared with laminar, because here the cars mainly travel in one lane and do not interfere with each other. Movement in the traffic congestion can be compared with the turbulent one, because here the cars interfere with each other and can often be reconstructed in traffic block. However, the movement in the traffic congestion is accompanied by a sharp decrease in the road's handling capacity (an analogue of the flow rate in the liquid), the maximum handling capacity is achieved in a traffic without jam movement. A contradiction arises, since the turbulent regime of fluid motion is achieved at a higher flow rate than laminar flow. As a result, the use of dependencies of the Reynolds number when describing traffic in a transport stream is possible only with the introduction of any assumptions.

From hydrodynamics, the continuity equation and the relationship between velocity and density are used; however, there are a number of drawbacks here too, due to which some researchers have abandoned continuous (gas and hydrodynamic models) models [9].

The conservation law of traffic flow of hydrodynamic models (an analogue-model) implies the use of the concepts of density and speed of cars moving along the road (pipeline); traffic flow must be continuous. When a traffic congestion occurs in front of an adjustable traffic light intersection, the flow of cars stops being uniform, but within the boundaries of the traffic jam itself, the flow can be considered uniform, but this model cannot take into account the uneven movement inside the traffic jam, that is, stops and speedups of cars.

The stochastic model given in [9] provides the calculation of the length of the queue of cars at the intersection with regulating traffic lights. The presence of moving obstacles in this model is not provided. The result of the modeling is the average queue length of cars accumulating at the intersection, taking into account the probability. In this case, we are not talking about minimizing the queue length - for this we need additional conversions. The object of calculation is the intersection, where the average speed of movement is small. This model cannot quantify the cycles of accelerations and stops when a car is moving in a traffic jam in front of an adjustable traffic light intersection either.

In the models of following the leader, the assumption is used that there is a connection between the head car and the one that follows it [10].

The theory of following the leader (microscopic model) involves the calculation of flow parameters depending on the car leader. In our case, the leader may be a truck driver. However, the theory of following the leaders implies the desire of all drivers to go as quickly as possible and does not imply overtaking. A truck driver may not strive to drive faster. Cars will tend to overtake the truck outside the jam. If, however, this model is used to describe the movement of cars in a traffic jam, then under certain assumptions it is possible (the truck will speed up and stop as intensely as cars, but in practice this is not likely to happen). The theory of following the leader can give an answer to the question about the length of the traffic block, however, you can use more simple dependencies to obtain the same result, moreover, in the problem considered in the work, the traffic block length will be one of the initial conditions, that is, already known.

In general, these methods are suitable for describing traffic flow without congestion. The theory taking into account the congestion was developed by Boris Kerner $[11,12]$. The flow phases are classified in it, besides it explains the reasons of congestion, that is, the theory explains the congestion. The three phase theory of Kerner is an alternative Theory of Traffic Flow, developed by Kerner. This theory focuses mainly on explaining the physics of the 
transition from free to dense flow resulting in space-time structures in dense traffic flow on highways. Kerner describes the three phases of a traffic flow, while classical theories based on the fundamental traffic flow diagram consider two phases: free flow and dense flow. According to the Kerner, two phases stand out in a dense stream: a synchronized stream and a wide moving cluster of machines (local congestion). Keren's theory describes well the movement of cars in a traffic jam well, but it is rather complicated. The authors of this article set themselves the task to offer a simple method for determining the number of cycles of cars stops and accelerations in a jam.

Every year more and more people are starting to use smartphones and various mobile applications, including navigation software applications [13]. This leads to increased accuracy of information provided by these applications on traffic jams. For example, the Yandex.Jams service in Yandex.Maps and Yandex.Navigator applications show users a picture of road congestion [14]. For this purpose, the service collects data on the workload of streets from different sources, analyzes them and displays them on Yandex.Maps. In the largest cities, where traffic jams are a serious problem, and not just a nuisance, the service calculates the point of traffic jams - the average level of traffic on the roads. The service technology is arranged in such a way that information about traffic jams is also collected by the users themselves.

Every few seconds the driver's device (most often a smartphone with one of the above applications installed) transmits its geographical coordinates, direction and speed to the Yandex.Probok computer system. Then, the analyzer builds a single route with information about the speed of passing of traffic jam - the track. The tracks come not only from private drivers, but also from the vehicles of Yandex partner companies (organizations with a large fleet of cars running around the city) [14].

In addition to their coordinates, drivers can provide the service with additional information about accidents, repair work on roads, or other road troubles.

GPS-receivers make some errors in determining the coordinates, which complicates the construction of the track. The error can "shift" the car a few meters in any direction, for example, on the pavement or the roof of the adjacent building. Coordinates coming from users fall on the electronic map of the city, on which all buildings, parks, streets with road markings and other urban objects are very accurately displayed. With these details, the program understands how the car actually moved.

In order to recreate the picture of the roads congestion correctly, it is necessary to check whether the track corresponds to the situation in its section. Yandex.Maps users can sometimes stop or slow down not because of traffic jams, but, for example, to buy something at the kiosk or not to miss a subtle turn. And if several more cars with mobile devices freely go by, such track will be screened out by the algorithm, because it does not reflect the actual traffic area. Therefore, the more users the service has, the more accurate the traffic information

After combining the tested tracks, the algorithm analyzes them and sets "green", "yellow" and "red" ratings to the corresponding sections of roads.

Next comes aggregation - the process of combining information. Every two minutes, the aggregator collects, as a mosaic, information received from Yandex.Maps mobile users in one scheme. This scheme is drawn on the Yandex.Maps "Jams" layer both in the mobile application and in the web service.

In Moscow, St. Petersburg and other major cities, Yandex.Probki service assesses the situation on a 10-point scale (where 0 points is "free" movement, and 10 points is "the city "gets stuck" in traffic jams). With this assessment, drivers can estimate how much time they will lose in traffic jams [14].

The scale of points is configured differently for each city: the situation that means a slight difficulty in Moscow, in another city means a serious traffic jam. For example, in St. 
Petersburg, with six points, the driver will lose approximately the same time as in Moscow at five.

Points are calculated as follows. Routes are drawn up in advance on the streets of each city, including major highways and avenues. For each route there is a reference time during which it can be driven along a free road without breaking the rules. After assessing the total workload of a city, the aggregator calculates how much the real time differs from the reference time. Based on the difference in all routes, traffic congestion in points is calculated.

It should be noted that for different roads (in the city and beyond the boundaries of the city) the same speeds correspond to the same colors that characterize the road congestion. For large roads, for example, the Moscow Ring Road (Moscow Ring Road), speeds are increased - the traffic jam is considered to be red to $20 \mathrm{~km} / \mathrm{h}$, and the Moscow Ring Road on Yandex.Maps will become green only at speeds from $50 \mathrm{~km} / \mathrm{h}$. And for narrow streets, for example, in the center, a traffic jam of up to $10 \mathrm{~km} / \mathrm{h}$ will be considered red, and at $25 \mathrm{~km} / \mathrm{h}$ and higher, the road will be considered to be free [15].

You can also view the average traffic speed in the traffic jam and the length of the jam in the Yandex.Maps web service. Any carrier may make the route, taking in consideration the congestion on roads.

Since the number of cycles of complete stops and speedups, deceleration and acceleration of the movement of freight road transport can significantly increase the cost of transportation, the authors of this article propose an easy way to take this into account, with the help of two quantities that can be obtained from the Yandex.Maps web service: the average speed of movement in the traffic jam and the length of the jam. Also, the Yandex.Maps web service displays information on whether the intersection of roads is regulated or not.

\section{Results}

Crossroads are regulated by traffic lights. Traffic lights must be divided into two types:

- Traffic lights operate in such a mode that do not cause congestion. It is assumed that if a car has fallen into the zone of action of such traffic light on an inhibit signal, then after changing the signal to an authorizing car, it leaves the zone of this intersection at the first acceleration. In this case, the probability of stopping at this intersection is as

$$
p_{i}=\frac{t_{o_{i}}}{t_{o_{i}}+t_{z_{i}}}
$$

$p i$ - is the probability to be in the regulated area of the $i$ - traffic light, during the prohibitory signal of this traffic light; $t_{o_{i}}$ is the duration of the enabling signal of the $i$ - traffic light per cycle, hour; $t_{z_{i}}$ - the duration of the prohibitory signal of the traffic light per cycle, hour.

Crossroads of this type on Yandex.Maps are determined as crossroads with installed traffic lights that do not cause congestion, that is, the color of the roads in the area of this intersection is green.

- Traffic lights, which due to their mode of operation and the intensity of the flow of vehicles in a certain period of daily time cause congestions.

Congestion - is an event in which to overcome the zone of action of a traffic light, the car is forced to carry out several cycles of a full stop and acceleration to a certain low speed, driving at that speed, followed by a full stop.

For traffic lights of the second type, there is a congestion of $l_{\text {prob }}$ length expressed in meters, indicated in red color in the Yandex.Maps service, and the indication of the average speed of movement in this traffic jam $V_{c p}$ is provided by the service in $\mathrm{km} / \mathrm{h}$. 
We have the following task: length of the traffic jam $\left(l_{\text {prob }}\right)$ and the average speed of the movement of vehicles in the jam $\left(V_{c p}\right)$ are known. It is also necessary to introduce the concepts: time of accelerated movement in one cycle after a full stop of a car while driving in a traffic jam $t_{p}$, time of the uniform movement in a cycle $t_{r d}$, after acceleration, time spent on deceleration to a full stop in one cycle $t_{T}$, speed with which the car will move in a traffic jam between acceleration and deceleration sections in a cycle $\left(V_{o p t}\right)$. It is necessary to derive the dependence on the given values of the number of complete cycles $(\mathrm{m})$ when the car is moving in a jam, that is, the number of complete stops and subsequent accelerations.

For clarity, it is necessary to build a graph of the dependence of speed on time. One cycle in Figure 1 is bounded by ACDFMN vertices. When solving the problem, we will assume that in the areas of acceleration and deceleration the car will move with constant acceleration. The acceleration modulus is taken into account as a straight line inclination tangent of the speed change eventually. Passable way of the car in Figure 1 looks like the area under the graph. The figures bounded by the straight lines which reflect cycles and the band under $V_{c p}$ should be equal, that is, their areas should be equal.

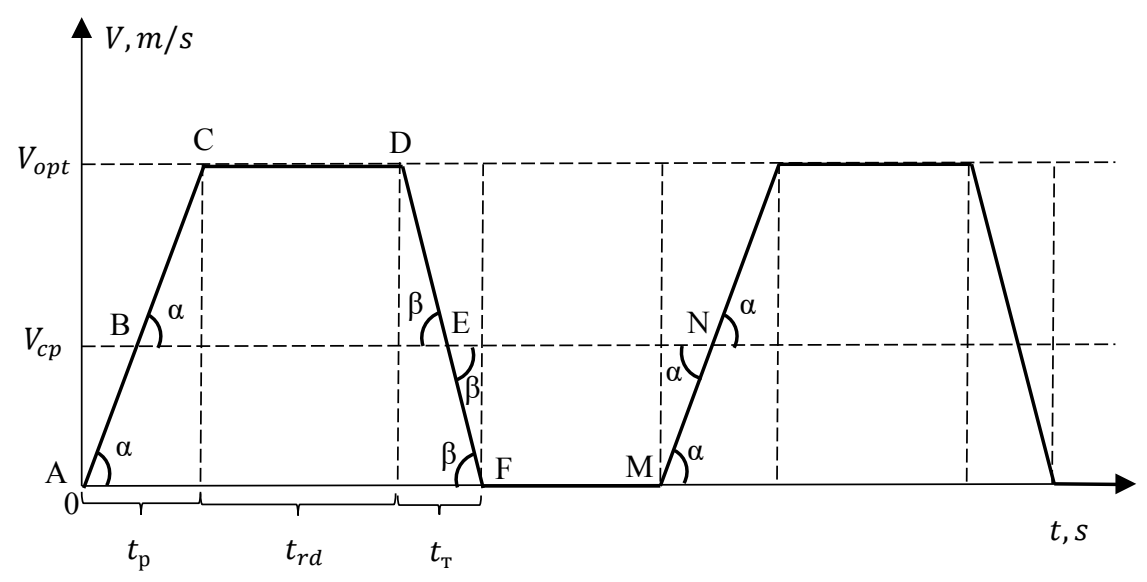

Fig. 1. The graph of the speed dependence on time. (2)

Using the graph in Figure 1, it is possible to work out a semantic system of the equations.

$$
\left\{\begin{array}{c}
S_{B C D E}=S_{E N M F} \\
m S_{A C D F}=l_{\text {prob }} \\
m\left(S_{A B E F}+S_{E N M F}\right)=l_{\text {prob }}
\end{array}\right.
$$

SBCDE, SENMF-are the areas of the corresponding figures in Figure 1.

Filling the system of the equations (2) we receive the system of the equations (3).

$$
\left\{\begin{array}{c}
\left(2 t_{o}-2\left(t_{p}+t_{T}\right)+\frac{V_{o p t}-V_{c p}}{V_{o p t}}\right)\left(V_{o p t}-V_{c p}\right)=\left(2 t_{z}+\frac{V_{c p}}{V_{o p t}}\left(t_{p}+t_{T}\right) V_{c p}\right. \\
m \frac{2 * t_{o}-t_{p}-t_{T}}{2} V_{o p t}=l_{\text {prob }} \\
\frac{m}{2}\left(2 t_{o}-t_{p}-t_{T}+\frac{V_{o p t}-V_{c p}}{V_{o p t}}\left(t_{p}+t_{T}\right)\right) V_{c p}+\left(2 t_{z}+\frac{V_{c p}}{V_{o p t}}\left(t_{p}+t_{T}\right) V_{c p}\right)=l_{\text {prob }}
\end{array}\right.
$$

The result of the solution of system of the equations (3) will be the dependence of quantity of full motion cycles of the car in traffic block from the corresponding sizes (4) 


$$
m\left(V_{c p}, V_{o p t}, t_{p}, t_{T}, l_{p r o b}\right)=\frac{2\left(V_{c p}-1\right)\left(V_{o p t}-V_{c p}\right) l_{p r o b}}{\left(V_{c p}-2\right)\left(t_{p}+t_{T}\right) V_{c p} V_{o p t}}
$$

Dependence (4) shows that the number of complete cycles is directly proportional to the length of the traffic block $\left(l_{\text {prob }}\right)$, and inversely proportional to the speed of uniform movement within the cycle $\left(V_{\text {opt }}\right)$. After reducing the dependence to the form shown in equation (5), it can be noted that the $m\left(V_{o p t}\right)$ graph is a hyperbole (Figure 2) with a horizontal asymptote (6).

$$
\begin{gathered}
m\left(V_{c p}, V_{o p t}, t_{p}, t_{T}, l_{\text {prob }}\right)=\frac{2\left(V_{c p}-1\right)}{V_{c p}-2} * \frac{t_{p r o b}}{t_{p}+t_{T}} *\left(1-\frac{V_{c p}}{V_{o p t}}\right) \\
\frac{2\left(V_{c p}-1\right)}{V_{c p}-2} * \frac{t_{p r o b}}{t_{p}+t_{T}}
\end{gathered}
$$

The particular interest is the ratio, which we call Par.

$$
\operatorname{Par}=\frac{t_{\text {prob }}}{t_{p}+t_{T}}
$$

Figure 2 shows the dependence of the number of cycles when a car is moving in a jam at certain average speed $\left(V_{c p}\right)$ and Par parameter on the speed of uniform movement in a cycle. The curves are plotted for $V_{c p}=7 \mathrm{~km} / \mathrm{h}$ and Par $=2$ (5 and 8). Determining the value of Par for the authors of the article is not possible. Freight carriers can do this by collecting statistics on the behaviuor of a car in a jam, as all trucks in the Russian Federation today are equipped with satellite tracking systems. The Par parameter shows how many times the time to overcome the entire jam by a car is more than the total time spent on acceleration and stopping in one cycle. From Figure 2 it can be seen that this parameter greatly influences the final result.

Automobile freight transport is one of the main air pollutants. But to abandon this type of transport for implementation of cargo transportation today is not possible. From this it follows that it is necessary to try to minimize in all possible ways the harmful effects on the ecology of this type of transport. The authors of this article have tried to draw attention to the increased fuel consumption of trucks in case of uneven movement. Moreover, at low speeds and accelerations, as well as idling, the process of burning fuel in internal combustion engines is the least effective, which leads to an increase in atmospheric air substances compared with the optimal operation of internal combustion engines. 


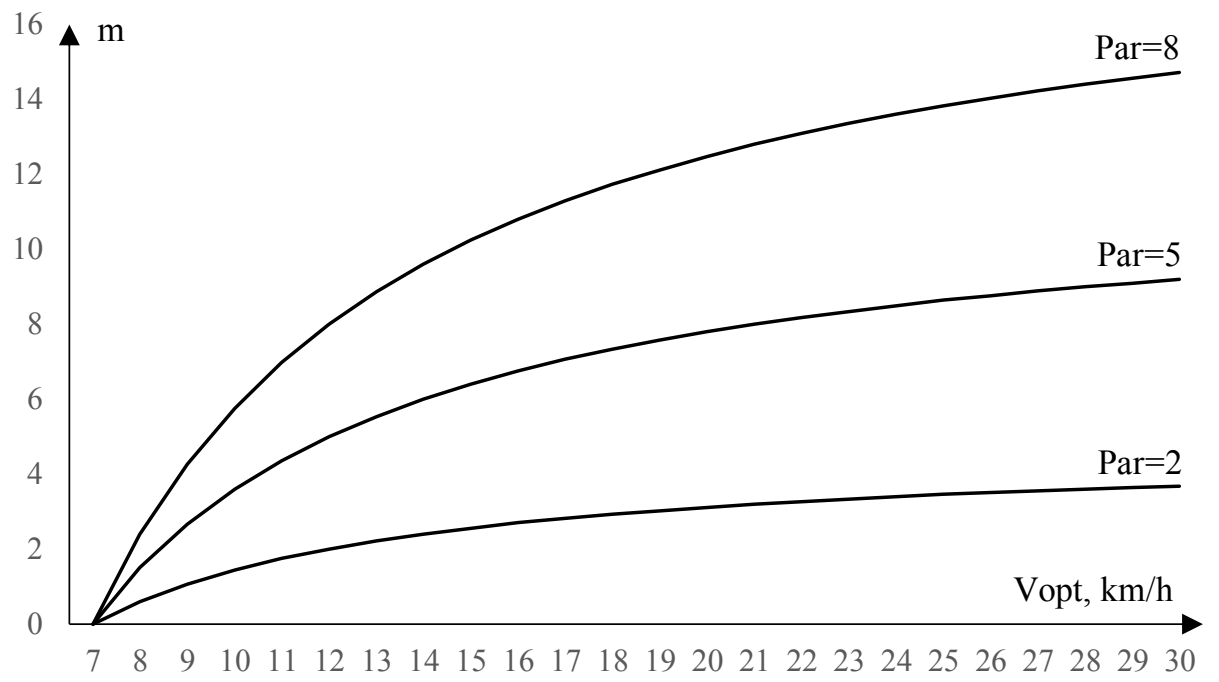

Fig. 2. Dependence $m$ on $V_{\text {opt }}$ at various Par.

\section{Discussion}

The paper proposes a method for calculating the number of cycles that consist of stopping a car before traffic jam, accelerating to a certain speed, driving at that speed in a jam, slowing down and stopping a car, staying in place until the beginning of the next cycle.

The theoretical foundations of existing models and techniques that also address the problems of the movement of vehicles in the traffic flow were considered. The strong and weak points of these models, including the difficulty in using some ones and the inability of each to answer the question posed in this work, are noted.

The presented method of calculation provides an answer to the question with the use of modern information systems on the example of "Yandex.Maps." With the help of this information system, it is possible to obtain basic data in the form of the average speed of a car in a jam and the length of the jam.

Also, it was revealed the need to consider and study additional parameters: acceleration time in one cycle, deceleration time in one cycle and some speed of uniform movement of the car within one cycle. These values need to be studied in detail by collecting statistics and processing tools statistics. This work can be done in conjunction with some carriers. Basic data can be obtained from carriers, since all trucks in the Russian Federation are equipped with the ERA GLONASS satellite tracking system and very many GPS systems to track vehicles in real time with the ability to record all driving parameters, including speeds with high frequency of recording all indicators. Most likely, these parameters will have a probabilistic nature and obey the law of the normal probability density distribution. Since we are talking about stochastic quantities, it is advisable to use the tools of simulation modeling, for example, on the platform "AnyLogistix".

After the implementation of all the above comments, it will be possible to obtain a technique that can quickly and simply answer the question posed in the paper. 


\section{Conclusions}

The paper describes the problem of air pollution by trucks. The relevance of the issues related to finding new ways for minimizing the negative impact of freight road transport on the environment, in particular, due to the possibility of using high-speed toll roads is proved. But the problem is that many freight carriers are reluctant to use toll roads, on which the movement of vehicles is more uniform, with a much smaller number of sections of braking, accelerating and stops. In these areas there is an increase in fuel consumption and in the amount of harmful emissions into the atmosphere. Many roads pass through settlements with regulated crossroads, which often form multi-kilometer jams. The authors of the article had an idea to determine the savings from using toll highways in comparison with ordinary roads, in order to clearly demonstrate to carriers that the charge for using highways does not always surpass these savings. In this regard, in this paper, the authors proposed the method for calculating the main indicator to determine the above-mentioned savings - the number of full stop cycles, the subsequent acceleration to a certain speed of the vehicle in the jam, the uniform movement with this speed and stop. Were also identified parameters that require further study. The nature of the functional dependence of the number of cycles on the rate of the speed of the section of the uniform movement of the car in a jam was determined. This dependence appeared to be inversely proportional. The dependence of the horizontal asymptote value on the additionally identified parameters was determined. Recommendations for further study of these parameters and improvement of the proposed methods are given.

\section{References}

1. A.A. Bavrin, D.A. Gavrilov, V.A. Dubolazov, N.S. Lukashevich, V.M. Makarov, Z.L. Simakova, E.R. Temirgaliev, Logistics 11 (2016)

2. V.A. Dubrovina, E.R. Temirgaliev, Science week SPbPU 234 (2016)

3. Russian vehicle fleet: main indicators (2018)

4. Fleet of trucks in Russia: key indicators (2016)

5. H.I. Pavlova, V.K. Novikov, Transport ecology 192 (2016)

6. Transport strategy of the Russian Federation for the period up to 2030 (2008)

7. E. Gladkih, Young Scientist 13.1, 33 (2019)

8. https://rtp.expert/news/682-mnenie_eksperta_svp_platon_i_platnye_dorogi_sedaiut _pribyl_gruzoperevozchikov (Last accessed 13.11.2018)

9. K.P. Mandrovskiy, Vestnik MADI 4, 32 (2014)

10. A.I. Pinyanskiy, A.P. Buslaev, T-comm: Telecommunications and transport 11, 27 (2017)

11. A.V. Gasnikov, S.L. Klenov, E.A. Nurmiskiy, Y.A. Holodov, N.B. Shamray, Introduction to mathematical modeling of traffic flows (2010)

12. S.L. Klenov, Trudi MFTI 2, 46 (2010)

13. Internet 2017-2018 in the world and in Russia: statistics and trends (2018)

14. https://yandex.ru/company/technologies/yaprobki (Last accessed 22.11.2018)

15. https://yandex.ru/blog/company/38031 (Last accessed 16.11.2018) 